\title{
La simulacion como herramienta para la mejora en el uso de recursos empresariales. Caso pruebas destructivas de calidad
}

Juan Victor Bernal ${ }^{1}$ y Mónica Belem Bernal Pérez ${ }^{2}$

\section{Resumen}

Creadores del éxito empresarial, los expertos coinciden en que los procesos de simulación son una herramienta que permite promover el desarrollo tecnológico y la sustentabilidad; a través de éstos, se permite fortalecer y generar nuevas capacidades y acciones que mejoran la eficiencia en la utilización de los recursos existentes, con una connotación de sentido social, económico y ambiental. En este marco, se presenta el trabajo que documenta la forma en que se aplica un modelo Montecarlo a las pruebas destructivas con las que se monitorea el nivel de calidad de la soldadura en una empresa metal mecánica de México, proponiendo ahorros considerables al reducir el nivel y costo del scrap, en beneficio de los grupos de interés, como lo son, trabajadores, accionistas, gobierno y sociedad.

Palabras clave: Simulación, calidad, pruebas destructivas.

1 Profesor de Tiempo completo de la carrera de Ingeniería en Logística y Líder de Línea de Investigación de Mejora en Procesos y Productos en el sector industrial. Tecnológico de Estudios Superiores de Cuautitlán Izcalli, Mexico.

2 Ingeniera en Gestión Empresarial y estudiante de Maestría. Tecnológico de estudios Superiores de Cuautitlán Izcalli, México. 


\begin{abstract}
Creators of business success, experts agree that the simulation processes are tools to promote technological development and sustainability; through them, it helps to strengthen and build new capabilities and actions that improve the efficient use of existing resources, with a connotation of social, economic and environmental sense. In this context, this paper documents how a Monte Carlo model is being applied to destructive tests for monitored the quality level of a welding in metal mechanics company on Mexico, offering considerable savings by reducing the level and cost applies occurs scrap the benefit of stakeholders, such as, employees, shareholders, government and society.
\end{abstract}

Key words: Simulation, quality, destructive tests. 


\section{Introducción}

Los expertos coinciden en que los procesos de simulación son una herramienta que permite promover el desarrollo tecnológico y la sustentabilidad; a través de éstos, se permite fortalecer y generar nuevas capacidades y acciones que mejoran la eficiencia en la utilización de los recursos existentes, con una connotación de sentido social, económico y ambiental, que pueden inducir al éxito empresarial. Hace posible conocer mejor un sistema y predecir su posible comportamiento bajo diferentes condiciones y circunstancias, incluso en aquellos tipos de situaciones que pueden ser complejos en su accionar.

Desde hace varios años, los procesos de simulación se han utilizado como una estrategia que permite conocer resultados a posibles escenarios que se desean someter a consideración. Son muchos los casos de aplicación que se encuentran, como los simuladores de vuelos para pilotos, aquellos usados para los negocios, e incluso los videojuegos hacen uso de ésta para generar situaciones virtuales variadas en los participantes.

Hay un gran número de programas de simulación para aplicaciones muy específicas, no obstante, el método Montecarlo se presenta como un algoritmo de fácil y efectivo modelaje, con resultados rápidos y confiables, que permite hacer juicios precisos en la toma de decisiones, mejorando sustancialmente los beneficios derivados de éstas.

En el área de calidad son variadas las aplicaciones, desde el análisis de fallas por cargas en materiales, hasta comportamientos de productos en su uso con los consumidores, lo que se conoce como tiempo de vida. La información que se obtiene de estos estudios permite una mejora de características de los productos y servicios, así como de los procesos de producción.

En términos sencillos, un modelo de simulación Montecarlo se construye a través de expresiones matemáticas con relaciones lógicas que permiten reproducir las condiciones reales del sistema en estudio, y que se opera mediante variables de entradas controladas y aleatorias, que dan un resultado o datos de salida, que es el propósito de esta técnica. Dicho resultado es contrastado contra un estándar o enfoque que guía la decisión a tomar.

La reproducción en gran cantidad de este modelo, con diferentes valores de entrada, genera igual número de resultados, en el espectro que se está estudiando, lo que permite tener un panorama más amplio para que el decisor pueda ir por la alternativa que mejores resultado esté proporcionando, desde distintas perspectivas. Mientras mejor se adecue el modelo al sistema real, mejorarán los resultados, lo que le da mayor confiabilidad al experimento de simulación.

La calidad se presenta no solo en la imagen de una empresa que es reconocida, sino en el nivel de satisfacción del cliente con el producto que se le ofrece y se aplica tanto al producto como a la organización. Uno de los requisitos que el cliente exige para 
asegurar la calidad de sus productos, es realizar una serie de pruebas que garanticen que las piezas cumplan con el resultado esperado. La forma de lograrlo es a través de un monitoreo sistemático que consta de ciertas pruebas realizadas, con el propósito de garantizar la conformidad con las especificaciones en el producto y que, además, es requerida por algunos clientes. Este proceso implica la participación continua de todos los trabajadores de una organización en la mejora en las etapas de diseño, desarrollo, fabricación y mantenimiento de los productos y servicios que ofrece de la organización.

En la presente entrega, se realiza una aplicación a las pruebas destructivas que una empresa del área automotriz aplica en sus procesos de control de calidad, para tomar la decisión que permita reducir sus costos de prueba sin perder el nivel de confianza actual en el monitoreo de su operación de soldadura, de forma tal que, con menos pruebas, se mantenga el estado de control establecido.

\section{Marco teórico}

\section{Simulación}

La simulación es el conjunto de relaciones lógicas, matemáticas y probabilísticas que integran el comportamiento de un sistema bajo estudio cuando se presenta un evento determinado (García, García \& Cárdenas, 2013). No es una técnica de optimización, sino que puede usarse para describir o predecir cómo operará un sistema con ciertas opciones dadas de los datos de entrada controlables y valores generados al azar de dichos valores de entrada controlables, que quizá conduzcan a sistemas deseables (Anderson, Sweeney \& Williams, 2011).

Se precisa conocer la manera como se comporta un sistema, considerado como un evento cuyas propiedades se desea considerar. La forma de hacerlo es mediante un experimento, que es el proceso de extraer datos de un sistema sobre el cual se ejerce una acción externa (Urquía \& Martín, 2013). Cualquier modelo de simulación tiene dos datos de entrada: controlables y probabilísticos, que se muestran esquemáticamente en la figura 1(Anderson, Sweeney \& Williams, 2011).

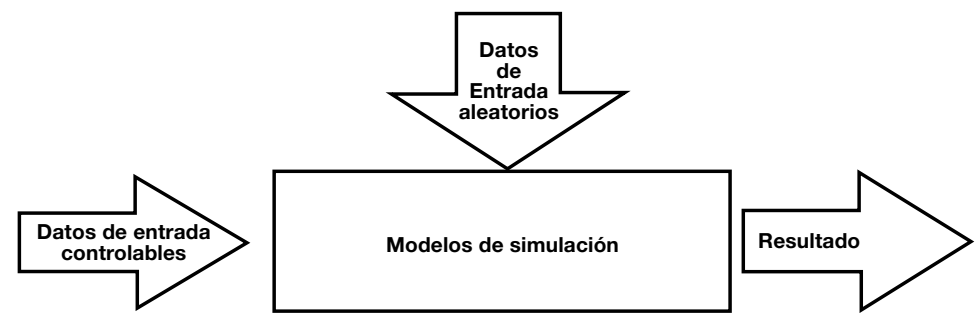

Figura 1: Esquema representativo de un modelo de simulación Montecarlo. Basado en Anderson, Sweeney \& Williams (2011). 
La simulación numérica de sistemas es una herramienta de estudio que permite cubrir el salto que se da entre la formulación teórica de un modelo y las comprobaciones experimentales, ya que permite evaluar la bondad de determinadas aproximaciones que se realizan en la formulación de sistemas o investigar sistemas cuya expresión analítica resulta demasiado compleja (Piñeiro, 2007). En términos generales, cualquier modelo de un problema de decisión empresarial podría denominarse un modelo de simulación, ya que representa o simula algunos aspectos del problema real (Bierman, Bonini \& Hausman, 2011).

Sirve para poder entender lo relacionado con un sistema real mediante la experimentación con el modelo que lo representa (Anderson, Sweeney \& Williams, 2009). Urquía \& Martín (2013) consideran que un modelo es una representación de un sistema desarrollado para un propósito específico; en el caso de la simulación por Montecarlo, se hace uso de uno matemático, relacionando las magnitudes de interés del sistema, se experimenta con él y se obtienen resultados. Con esto, se intenta conocer la estructura de los sistemas de estudio y obtener inferencias sobre su comportamiento.

Los modelos de simulación tienen dos características importantes a considerar: evalúan diversas alternativas propuestas y se toma una decisión con base en la comparación de resultados, y, por otra parte, el sistema se estudia mientras opera durante el tiempo y se incluyen los efectos de los resultados en el siguiente (Bierman, Bonini \& Hausman, 2011).

\section{Números aleatorios}

Para poder realizar una simulación que incluya variabilidad dentro de sus eventos, es preciso generar una serie de números que sean aleatorios por sí mismos, y que su aleatoriedad se extrapole al modelo de simulación que se está construyendo (García, García \& Cárdenas, 2013); debido a la complicación en la generación de éstos números y su comprobación de que realmente tiene esta condición, se utilizan números que pueden disponerse de una tabla o bien, generarlos por métodos ya determinados en una computadora, a los que se le llama pseudoaleatorios, pero que son confiables para emplearse en las aplicaciones Montecarlo.

Existen diferentes métodos de generación de números pseudoaleatorios; García, García \& Cárdenas (2013) explican algunos algoritmos como el cuadrado medio, productos medios, multiplicador constante, lineal, congruencial multiplicativo, congruencial aditivo, entre otros, que parten de un número semilla, y a través del procedimiento que señala cada método, se obtiene una serie de valores que se pueden utilizar para simulación.

Los números pseudoaleatorios generados por computadora, se obtienen del empleo de fórmulas matemáticas, no son técnicamente aleatorios, sin embargo su 
principal diferencia con los aleatorios puros es principalmente filosófica, y se utiliza el término de "número aleatorio" independientemente de si son o no generados por una computadora (Anderson, Sweeney \& Williams, 2011).

Cuando se generan por computadora, son decimales comprendidos en el intervalo $(0,1)$, que excluye ambos límites, es decir, el cero y el uno. Tienen una distribución de probabilidad uniforme, aunque en algunos modelos se pueden precisar otro tipo de distribuciones que se ajusten al comportamiento esperado. Para este trabajo, se hace uso de aquellos que están uniformemente distribuidos.

\section{Ensayos destructivos en el control de calidad}

Las propiedades mecánicas tanto de metales base como de uniones soldadas deben ser verificadas con el objeto de asegurar la calidad del conjunto respecto a su diseño. Los ensayos a efectuar dependerán de cada caso en particular. Los que normalmente se aplican son: el ensayo de resistencia a la tensión; ensayo de resistencia al doblez; ensayo de Nick-Break; ensayo de resistencia al impacto; y el ensayo de dureza (Ensayos destructivos, s. f.).

Un ensayo o prueba de tensión consiste en estirar una probeta o muestra de material a probar, hasta su rompimiento, en una maquina especial y los resultados obtenidos nos proporcionan una cantidad de información importante, como la resistencia a la tensión máxima, el esfuerzo de cedencia o ruptura, el porcentaje de elongación, y el de reducción de área. Algunos de estos valores pueden ser determinados de la probeta mediante un calibrador, mientras que otros pueden ser cuantificados solamente mediante el análisis del diagrama de esfuerzo-deformación el cual es obtenido durante el ensayo.

Mediante este ensayo se efectúa la calificación de procedimientos de soldadura. El objetivo de dicho ensayo es el de verificar el comportamiento de la unión soldadura con respecto al metal base, debiendo ser igual o mejor que el metal base. Se podrá desprender la base, pero no la soldadura, ya que si esto llega a ocurrir, se cuestiona el proceso de unión.

El ensayo de Nick-Break es usado principalmente en la industria petrolera, para la calificación de los procedimientos y soldadura de tuberías de conducción. Este método evalúa la sanidad de la soldadura, mediante la posible presencia de discontinuidades en la superficie de fractura del espécimen ensayado. La fractura se localiza en la soldadura mediante 2 o 3 ranuras a lo largo de la superficie.

La prueba de ruptura de filete, o de desgarre, se verifica que la soldadura muestre una apariencia superficial satisfactoria además inspecciona la superficie fracturada para asegurarse que la soldadura presenta evidencia de fusión en la raíz y no muestra áreas des de fusión incompleta de la soldadura con el metal base o porosidades en la soldadura. 
En cuanto a la prueba de dureza, este concepto se puede definir como la resistencia al desgaste, rayado, penetración, indentación, maquinabilidad y capacidad de corte; se utilizan casi 30 pruebas para medirla, siendo las más comunes la Brinell, Vicker, y Rockwell (Bawa, 2007). En cuanto al equipo de ensayo, este deberá estar debidamente calibrado, de lo contrario, obviamente, los resultados obtenidos no tienen ninguna validez.

\section{Pruebas de hipótesis}

Varios problemas de ingeniería requieren que se tome une decisión entre aceptar o rechazar una proposición sobre algún parámetro. Esta proposición recibe el nombre de hipótesis, y el procedimiento de toma de decisión se conoce como prueba de hipótesis. Para validar la información, Montgomery \& Runger (2013) proponen un procedimiento de ocho pasos que se lista como: definición de la prueba, establecimiento de la hipótesis nula y alternativa, selección del nivel de significancia, definición del estadístico de prueba, determinación del criterio de rechazo, realización de los cálculos y enunciado de la conclusión.

\section{MARCO CONTEXTUAL}

\section{La empresa}

Dentro de la industria automotriz mexicana, una empresa dedicada a la manufactura de bastidores para asientos de automóviles, sigue normas estrictas en su operación. Es un proveedor de clase mundial de mecanismos avanzados y conjuntos unidos por soldadura. Su liderazgo internacional la llevan a que la calidad y la mejora continua sean consideradas como importantes factores que le permiten lograr calidad total así como la satisfacción del cliente.

Para este proceso, las partes tubulares de fierro, son cortadas y conformadas en diferentes dimensiones, y unidas mediante soldadura que aplican una línea de cinco robots, que conforman la estructura del bastidor, que es la base para la fabricación de un asiento para automóvil [ver figura 2]. 

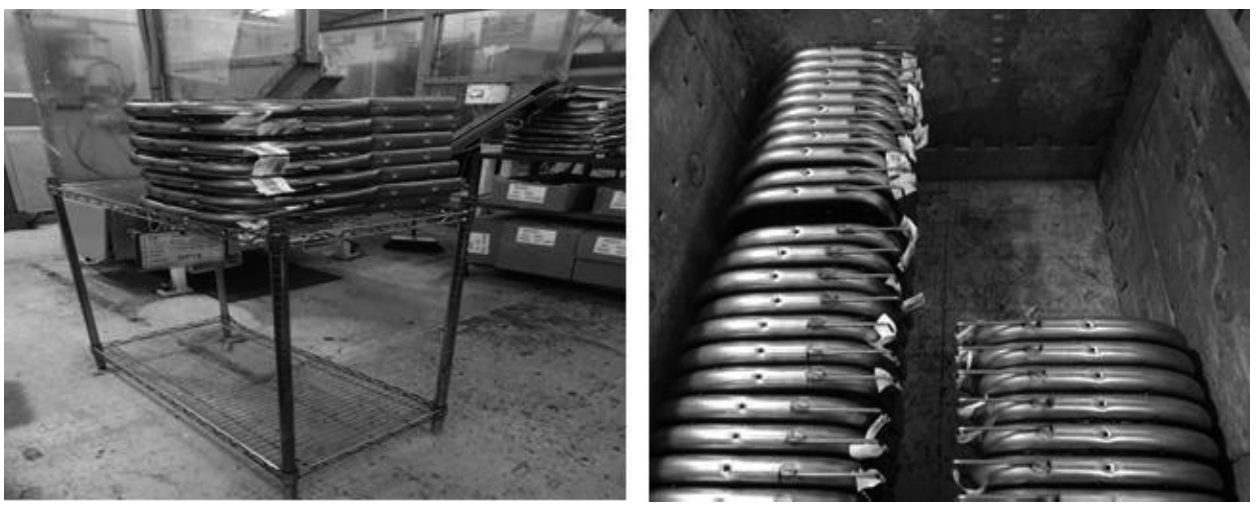

Figura 2. Muestra de partes de bastidores saliendo del proceso de soldadura, para manufacturar el asiento de un automóvil.

Para garantizar el cumplimiento con los requisitos de calidad, los productos son sometidos a pruebas tanto destructivas como no destructivas. Dentro de las primeras, se aplican aquellas de tensión a las soldaduras de dichos bastidores, y que los destruyen por su naturaleza, lo que genera un material de desecho o scrap.

\section{Descripción de las pruebas}

La empresa maneja dos tipos de pruebas denominadas "Cut and Etch" y "Tear Down" o Prueba de desgarre. La primera, a través de un corte que se realiza en la soldadura; no determina si existe fusión mide únicamente el cordón efectivo, se emplea un calibrador para realizar dicha medición, registrando y comparándola contra las especificaciones establecidas. Se conforma por medición de pierna, es decir, el ancho de la soldadura, la profundidad de fusión y la garganta. Este método no determina si existe fusión, únicamente mide el cordón efectivo, empleando un calibrador para realizar esta medición, que se registra para control y monitoreo del proceso.

La prueba de "tear down" o prueba de desgarre, consiste en tomar una pieza con proceso de soldadura de micro alambre, para tratar de separar las uniones entre componentes por cordones de soldadura. Se realiza con ayuda de la maquina universal de ensayos, cincel neumático o con cincel manual y martillo, midiendo la resistencia a la tensión aplicada. La prueba destructiva se realiza con ayuda de la maquina universal de ensayos, cincel neumático o con cincel manual y martillo, tratando de separar las diferentes uniones en el ensamble.

El procedimiento de la prueba de penetración de desgarre es el siguiente. El cordón de soldadura que será analizado deberá ser cortado transversalmente con ayuda del cortador de disco, de modo que se observen las partes soldadas y la unión 
de soldadura. La frecuencia y cantidad de cordones a ser analizados deberá realizarse de acuerdo con el plan de control. Una vez realizado el corte transversal al cordón de soldadura, se deberá pulir perfectamente la pieza antes de ser atacada con una sustancia química. Se debe revisar que la sección del cordón de soldadura analizado no presente, porosidad, fractura, falta de continuidad, doble cordón, poro o túnel.

Se realizan cuatro pruebas diarias a cada robot, tomando una pieza por turno de cada uno de sus cuatro modelos, lo que se dispone de información para un total de 20 ensayos diarios, muestras que ya no se pueden utilizar posteriormente. El estudio consideró una producción de seis meses, registrando el resultado de forma discreta, es decir como pasa o no pasa, para su tratamiento de forma estadística. El criterio para evaluar la soldadura con este método, es de la forma siguiente:

No aceptable (No pasa). Cuando al realizar esta prueba destructiva, el cordón de soldadura se desprenda de cualquier componente con el esfuerzo aplicado entre la unión de soldadura, este problema es originado por falta de fusión y penetración de soldadura, conocido como "soldadura fría"

Prueba aceptada (si pasa). El ensamble debe presentar desgarre de material en componentes (generalmente se desgarra la lámina) o presenta una deformación excesiva, unión abierta más de $5.0 \mathrm{~mm}$, sin tener desprendimiento de cordones de soldadura o cordones fracturados por el esfuerzo aplicado [ver figura 3].

Los resultados de las pruebas se llevan como registros de evidencia que respaldan el sistema de calidad, por lo que se deben llevar de forma organizada.
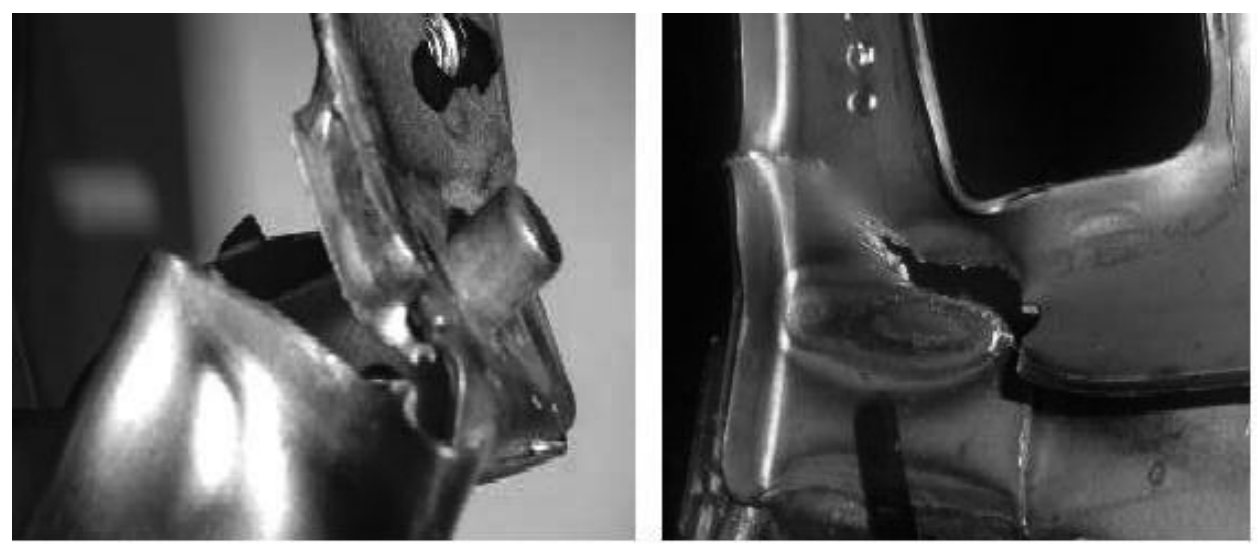

Figura 3. Ensamble deformado y con desgarre de material (lámina), sin presentar desprendimiento o fractura en cordones de soldadura, resultado aceptable de la prueba destructiva.

Cuando un bastidor se somete a las pruebas, es destruido, no puede ser reutilizado, por lo que se desecha. Los costos calculados solamente por desperdicio de material ascienden a $\$ 256,376$ USD anuales. Para mejorar la aplicación de 
los recursos y reducir los costos, se propone solo efectuar pruebas a 10 de las estructuras, con lo que se espera tener ahorros del $50 \%$ de entrada, sin perder sensibilidad en el monitoreo del proceso.

\section{Desarrollo}

\section{Metodología}

Para el desarrollo de este trabajo se utiliza una metodología hipotética experimental, que consiste en generar una hipótesis nula que será probada a través de un modelo de simulación Montecarlo, con el propósito de rechazar o no rechazar su validez. Dicho modelo se crea a partir de los datos históricos obtenidos en el tiempo de estudio. Su validez es a través de una prueba estadística de hipótesis para proporciones.

\section{Hipótesis Nula}

La hipótesis nula que se formula es la siguiente: la proporción de la prueba simulada con diez pruebas destructivas es igual al valor de la proporción histórica obtenida en los seis últimos meses.

\section{Análisis de los datos}

En el proceso de soldadura se cuenta con 5 robots que realizan la operación. Por cada uno, se toman cuatro piezas para formar la muestra diaria de veinte especímenes. Se hace el registro para monitorear el proceso y se recaban los datos de los seis meses anteriores al presente estudio. En este espacio de tiempo se realizaron 7496 pruebas destructivas, 5340 de "tear down" y 2156 de "cut and etch". El número de aquellas que no pasaron el requisito de calidad ascendió a 317 , lo que da un porcentaje del $4.23 \%$.

En esta propuesta, se considera que, al tener cinco robots con niveles de producción idénticos, presentan una distribución de probabilidad distribuida de forma uniforme para seleccionar una muestra completamente aleatoria. Esto permite generar un primer modelo para simular el tipo de celda de soldadura del que se tomará el bastidor a probar.

Cada robot tiene un $20 \%$ de ser seleccionado, por lo que se generan cinco intervalos a considerar, uno para cada robot. La probabilidad acumulada limita cada uno de dichos intervalos, teniendo una longitud de 0 a 1 , sin tocar los extremos. Así, el robot 1 estará definido desde 0.0001 hasta el valor de 0.1999; el 2 desde 0.2000 hasta el 0.3999 , el 3 de 0.4000 al 0.5999 , el 4 de 0.6000 al 0.7999 , y el 5 de 0.8000 hasta 0.9999 [ver 
figura 4]. Esto permite que las fronteras de cada espacio no se traslapen al momento de usar este primer modelo al definir de qué celda de soldadura se tomará la probeta. Con los resultados históricos por cada robot, se obtienen los porcentajes de falla, que sirven de base para generar el segundo modelo, que dará el porcentaje de la prueba.

\begin{tabular}{|c|c|c|}
\hline Robot & \multicolumn{2}{|c|}{ Intervalo } \\
\hline 1 & 0.0001 & 0.1999 \\
\hline 2 & 0.2000 & 0.3999 \\
\hline 3 & 0.4000 & 0.5999 \\
\hline 4 & 0.6000 & 0.7999 \\
\hline 5 & 0.8000 & 0.9999 \\
\hline
\end{tabular}

Figura 4. Modelo Montecarlo para simular la selección del robot del que se toma el espécimen para la prueba destructiva.

En el segundo modelo, se simula el resultado de la prueba. Para cada robot se genera un modelo dependiendo de su porcentaje de falla histórico por los resultados anteriores, que se usa dependiendo del robot seleccionado en la primera simulación. Por ejemplo, si se obtiene que el robot 1 es aquel del que se va a tomar la muestra, la segunda simulación proporciona el resultado de la prueba, es decir, pasa o no pasa [ver figura 5].

\begin{tabular}{|c|c|c|}
\hline \multicolumn{3}{|c|}{ MODELO ROBOT 1 } \\
\hline Resultado de la prueba & \multicolumn{2}{|c|}{ Intervalo } \\
\hline Pasa & 0.0001 & 0.9719 \\
\hline No Pasa & 0.9720 & 0.99999 \\
\hline
\end{tabular}

Figura 5. Modelo Montecarlo para simular el resultado de la inspección. Se muestra el modelo para el primer robot.

Para que se pueda trabajar en estos modelos, se generan números aleatorios, dos por cada ciclo, que deben ser utilizados para simular un tipo de robot y el resultado de la prueba, respectivamente. Por ejemplo, si para simular la selección del robot se obtiene el 0.0154 , que se encuentra en el intervalo del robot 1 , entonces se toma el Modelo Robot 1; si un segundo número que se obtiene es el 0.56275 y se emplea en el segundo modelo, el resultado es una prueba que si pasa.

Se realizan, entonces, la cantidad de 1,680 ciclos para simular 10 pruebas diarias, durante los próximos seis meses. El registro de los datos permite ver el comportamiento simulado, tanto en el robot seleccionado como en el resultado de la prueba [ver figura 6]. 


\begin{tabular}{|c|c|c|c|c|}
\hline \multicolumn{5}{|c|}{ MES 1} \\
\hline PRUEBA & RANDOM & ROBOT & RANDOM & RESULTADO \\
\hline 1 & 0.10163505 & 1 & 0.09424896 & 0 \\
\hline 2 & 0.45247968 & 3 & 0.83678298 & 0 \\
\hline 3 & 0.54491625 & 3 & 0.1607889 & 0 \\
\hline 4 & 0.03991656 & 1 & 0.88663922 & 0 \\
\hline 5 & 0.72098635 & 4 & 0.59745391 & 0 \\
\hline 6 & 0.37108624 & 2 & 0.42779304 & 0 \\
\hline 7 & 0.56756663 & 3 & 0.17401353 & 0 \\
\hline 8 & 0.07582962 & 1 & 0.92835165 & 0 \\
\hline 9 & 0.76503296 & 4 & 0.59451088 & 0 \\
\hline 10 & 0.60627256 & 4 & 0.44134919 & 0 \\
\hline 11 & 0.94632942 & 5 & 0.11151678 & 0 \\
\hline 12 & 0.34934595 & 2 & 0.34821634 & 0 \\
\hline 13 & 0.33530855 & 2 & 0.15328252 & 0 \\
\hline 14 & 0.923871 & 5 & 0.39533335 & 0 \\
\hline 15 & 0.73574063 & 4 & 0.86041 & 0 \\
\hline 16 & 0.91496982 & 5 & 0.16300184 & 0 \\
\hline 17 & 0.85172343 & 5 & 0.86654642 & 0 \\
\hline 18 & 0.74724309 & 4 & 0.59062217 & 0 \\
\hline 19 & 0.41943833 & 3 & 0.89153032 & \\
\hline
\end{tabular}

Figura 6. Vista parcial de los resultados simulados para las pruebas destructivas.

\section{Resultados y conclusiones}

Al analizar los resultados obtenidos en las pruebas destructivas simuladas, se obtiene un porcentaje de falla del $3.282 \%$, que se compara estadísticamente con el histórico de $4.23 \%$. A través de una prueba de hipótesis de proporciones, con un nivel de significancia del 5\%, no se rechaza la hipótesis nula, por lo que se concluye que no hay diferencia significativa entre ambas proporciones, y es válido reducir el número de pruebas.

Es importante aclarar que la simulación no sustituye las pruebas de calidad que se aplican en los procesos, lo que nos permite obtener es experiencia de posibles escenarios que se dan, dependiendo de la precisión del modelo.

Esta herramienta se puede aplicar a cualquier tipo de industria con problemas e incertidumbres en su proceso de toma de decisiones, mejorando los resultados al incluir una experiencia que les permita comprender y dominar las situaciones complicadas, con beneficios para los grupos de interés, en los que se incluyen empleados, empresarios, gobierno, sociedad y el país que los envuelve. 


\section{Referencias}

Anderson, D., Sweeney, D. \& Williams, T. (2011). Métodos cuantitativos para los negocios. $11^{\mathrm{a}}$ edición, México: Thompson editores. Pág. 650-675.

Bawa, H. S. (2007). Procesos de Manufactura. México: Mc Graw Hill Interamericana Editores.

Bierman, H., Bonini, C, \& Hausman, W. (2011). Análisis cuantitativo para la toma de decisiones. México: McGraw-Hill Interamericana.

García, E., García, H., \& Cárdenas, L. (2013). Simulación y Análisis de Sistemas con Promodel. $2^{\mathrm{a}}$ edición, México: Pearson, Educación.

Ensayos destructivos (s. f.). En Supervisión de estructuras. Recuperado de https:// sites.google.com/site/supervisiondeestructuras/estructuras-de-acero/tema-7ensayos-destructivos

Montgomery, D. \& Runger, G. (2013). Probabilidad y Estadística aplicadas a la ingeniería. México: Editorial Limusa S.A. de C. V.

Piñeiro, Y. (2007). Simulación de Montecarlo de Sistemas complejos de red. Tese de Doutoramente. Faculatde de Física. Universidad de Santiago de Compostela, España. Pág. 15-17.

Urquía, A. \& Martín, C. (2013). Modelado y simulación de eventos discretos. Madrid: Universidad Nacional de Educación a Distancia. 
